\title{
How to Achieve Efficiency and Accuracy in Discrete Element Simulation of Asphalt Mixture: A DRF-Based Equivalent Model for Asphalt Sand Mortar
}

\author{
Yu Liu, ${ }^{1}$ Peifeng Su $\mathbb{D}^{1},{ }^{1}$ Miaomiao Li, ${ }^{1}$ Hui Yao $\mathbb{D}^{2},{ }^{2}$ Junfu Liu, ${ }^{2}$ Mei Xu, ${ }^{2}$ Xiaodong Zhou, ${ }^{3}$ \\ and Zhanping You $\mathbb{D}^{3}$ \\ ${ }^{1}$ Highway School, Chang'an University, South Erhuan Middle Section, Xi'an, Shaanxi 710064, China \\ ${ }^{2}$ Beijing Key Laboratory of Traffic Engineering, College of Metropolitan Transportation, Beijing University of Technology, No. 100, \\ Pingleyuan, Chaoyang, Beijing 100124, China \\ ${ }^{3}$ Department of Civil \& Environmental Engineering, Michigan Technological University, 1400 Townsend Drive, Houghton, \\ MI 49931, USA \\ Correspondence should be addressed to Hui Yao; huiyao@mtu.edu
}

Received 24 June 2020; Revised 18 November 2020; Accepted 7 December 2020; Published 22 December 2020

Academic Editor: Fan Gu

Copyright $\odot 2020 \mathrm{Yu}$ Liu et al. This is an open access article distributed under the Creative Commons Attribution License, which permits unrestricted use, distribution, and reproduction in any medium, provided the original work is properly cited.

\begin{abstract}
The clump-based discrete element model is one of the asphalt mixture simulation methods, which has the potential to not only predict mixture performance but also simulate particle movement during compaction, transporting, and other situations. However, modelling of asphalt sand mortar in this method remains to be a problem due to computing capacity. Larger-sized balls (generally $2.0-2.36 \mathrm{~mm}$ ) were usually used to model the smaller particles and asphalt binder, but this replacement may result in the mixture's unrealistic volumetric features. More specifically, replacing original elements with equal volume but larger size particles will increase in buck volume and then different particle contacting states. The major objective of this research is to provide a solution to the dilemma situation through an improved equivalent model of the smaller particles and asphalt binders. The key parameter of the equivalent model is the diameter reduction factor (DRF), which was proposed in this research to minimize the effects of asphalt mortar's particle replacement modelling. To determine DRF, the DEM-based analysis was conducted to evaluate several mixture features, including element overlap ratio, ball-wall contact number, and the average wall stress. Through this study, it was observed that when the original glued ball diameters are ranging from $2.00 \mathrm{~mm}$ and $2.36 \mathrm{~mm}$, the diameter reduction factor changes from 0.82 to 0.86 for AC mixtures and 0.80 to 0.84 for SMA mixtures. The modelling method presented in this research is suitable not only for asphalt mixtures but also for the other particulate mix with multisize particles.
\end{abstract}

\section{Introduction}

In the past decades, the discrete element method (DEM) has been popularly used for simulating multisize particulate mixtures, such as asphalt mixtures, sands, graded stones, and chemically bounded mixtures [1]. In the simulated model, the discrete elements are applied to represent different components in the mixture. From a simple two-dimensional ideal model [2] to a complex three-dimensional image-based model [3], the DEM model is more and more similar to the real mixture, and the simulation results are more credible
[4-6]. Currently, there are two main types of discrete element models which were popularly used for simulating asphalt mixtures in the past decades: the cluster-based models $[7,8]$ and the clump-based models [9]. The primary goal of these DEM models was to predict mechanical behaviours. Furthermore, with the development of the clumpbased model, it became practicable to apply DEM in other studies, for instance, the compaction stage.

In the cluster-based models, both coarse aggregates and asphalt sand mortar are simulated through mono-sized balls which are bounded through contact bonding models [10]. 
The coarse aggregates and the asphalt sand mortar are distinguished by assigning different stiffnesses and strength properties [11]. After deleting part of balls randomly, asphalt mixture specimens with certain air voids can be obtained [12]. The cluster-based models have the following advantages: (1) coarse aggregate morphological features can be realistically simulated with accurate images of mixtures or coarse aggregates [13]; (2) the volumetric features of coarse aggregates, asphalt sand mortar, and air voids can be modelled with high accuracy [14]. The disadvantages of these models are also obvious: (1) only compacted specimens can be simulated, which means the problems related to the compaction process, segregation, and packing cannot be analysed, and the simulation model applicability is limited by this way of modelling; (2) a large number of elements are needed to ensure reasonable accuracy of simulating asphalt sand mortar; hence, the computing efficiency is relatively low.

The clump-based model is an updated model compared to the cluster-based model, with higher computational efficiency and more possible applications. In the clump-based models, the coarse aggregates are simulated by clumps with realistic particle shapes and sizes [15]. The use of the clump element tremendously increases the computing speed since pebbles within the same clump will not form contact. For fine aggregates, fillers and asphalt are integrally simulated as asphalt sand mortar in two ways for different research goals: (1) mono-sized balls, which are the same as the cluster-based model, can be used for the purpose of mechanical behaviour prediction; (2) equivalent ball elements with same or similar diameters for the studying of particle movements during compaction or other stages. In this way, the number of ball elements was determined by dividing the total volume of asphalt sand mortar by the ball volume.

The use of equivalent ball elements way is a compromise to ensure practical computational efficiency. Researchers mostly replace fine particles $(0-2.36 \mathrm{~mm})$ and asphalt with larger size particles (generally $2.0-2.36 \mathrm{~mm}$ ) since it is unrealistic to generate fine particles with real size and number [9]. Different from the first way, these particles still retain particular properties during compaction. However, this compromise definitely leads to the volumetric problem since larger size balls actually occupy more space in the specimen.

In sum, although the clump-based model provides a way to study particle movements during the compaction stage, the challenging task is to find a potential solution to this dilemma situation: researchers prefer selecting larger size balls ( $2 \mathrm{~mm}$ or larger) to build the equivalent models of asphalt sand mortar for the higher computational efficiency. However, this method is usually empirical and lacks quantitative analysis and verification, which will lead to the following problems: (1) since the ball-ball interactions in the equivalent models are different from those in the asphalt sand mortar, particle-interlocking effects may result in unrealistic mechanical responses. (2) The equivalent model of ball elements has a larger volume than realistic asphalt sand mortar of the same mass. In other words, the equivalent model cannot realistically simulate asphalt sand mortar since they have different volumetric features. (3) With the increasing ball element diameters of the equivalent model, the particle-interlocking effects and volumetric discrepancy become more serious.

Based on the above discussion, the major objective of this research is to provide a potential solution to the dilemma situation through an improved equivalent model of the smaller particles and asphalt binders for the clumpbased model. Hence, the asphalt sand mortar can be well simulated during the compaction stage without causing the volumetric problem. In order to minimize the effects of particle interlocking, a key parameter: diameter reduction factor (DRF) is developed to reduce the total volume of the original equivalent ball element model. By proportionally reducing the mortar particle diameter, the DRF method can reduce the interfering caused by replacing asphalt sand mortar with larger particles. Optimum DRF values could minimize the buck volume discrepancy before and after replacing. The DRF value is determined through analysing the overlap ratio of mortar particle and average wall stress, ensuring the volumetric feature of asphalt sand mortar and particle interaction between mortar ball element and coarse particles. The details of the DRF definition and value determination will be introduced in Section 2. Suitable DRFs are recommended for different mixture gradations, and the results are verified in lab tests.

\section{An Improved Equivalent Model for Simulating Asphalt Sand Mortar}

2.1. Volumetric Testing of Coarse-Grained and Fine-Grained Mineral Mixtures. The bulk volume of the small-sized particle mixture may be different from a large-sized particle mixture. Even though with the same mass, the difference still occurs in terms of particle-interlocking actions and volumetric features. In order to verify this postulate, an experimental test was conducted; two mineral mixtures that have the same mass were put into the two identical cups, as shown in Figure 1. Mix A is fine-grained with particles from $0.00 \mathrm{~mm}$ to $2.36 \mathrm{~mm}$, while the mix B is coarse-grained with particles from $1.18 \mathrm{~mm}$ to $2.36 \mathrm{~mm}$. Both of the two mixtures are stacking conditions without compaction; therefore, the packing energy can be assumed to be equal. The bulk volume of mix A and mix B is $85 \mathrm{ml}$ and $100 \mathrm{ml}$, respectively. The volumetric difference is about $17.9 \%$.

Through this experimental test, it could conclude that the same mass of mixtures may have significantly different volumes. With this conclusion, the authors believe that the larger-sized rigid balls for modelling asphalt mortar may occupy the larger volume in a discrete element model. Therefore, in order to improve the discrete element model accuracy, it is necessary to determine the optimum sizes of rigid balls.

2.2. Clump-Based DEM Asphalt Mixture Model Establishment. In this research, the clump-based DEM model of asphalt mixture was built by the following procedures: 


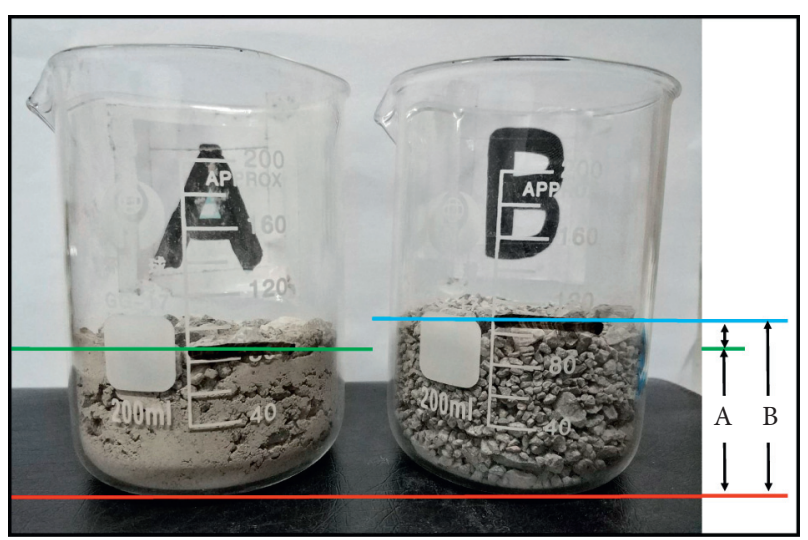

FIGURE 1: Volumetric testing of coarse-grained and fine-grained mixtures.

(1) Coarse aggregates morphological features were obtained through 3D scanning and image processing. Then, virtual sieving tests were carried out to obtain clump templates in PFC [16].

(2) Coarse aggregates (modelled by clump elements) were generated according to the gradation and clump templates, as shown in Figure 2(a).

(3) Asphalt sand mortar (modelled by ball elements) was generated, as shown in Figure 2(b).

(4) The specimen was compacted with hydrostatic pressure, as shown in Figure 2(c).

Note that different colours in Figure 2 represent different particle size groups. Through the above steps, a virtual DEM model with a real particle shape can be built. Parameters used in virtual models are listed in Table 1 [17].

\subsection{Diameter Reduction Factor for Modelling Asphalt Sand} Mortar. In most of the existing studies, the glued rigid balls of diameters from $2.00 \mathrm{~mm}$ to $2.36 \mathrm{~mm}$ were commonly used to build the equivalent model of asphalt sand mortar. The number of the rigid balls was determined through the following two steps: (1) in the first step, the volume of asphalt sand mortar is calculated from weights and densities of fine aggregates, fillers, and asphalt binder according to the mixture design; (2) in the second step, users may select diameters of the rigid balls (ranging from $2.00 \mathrm{~mm}$ to $2.36 \mathrm{~mm}$ for instance); the number of the rigid balls is calculated through dividing the total volume of asphalt sand mortar by the individual ball's volume. However, this approach generally ignores the real volume occupied by mortar particles; similar to Figure 1, larger size particles with the same mass will occupy more volume in the specimen and, therefore, change the mixture specimen skeleton and further mechanical performance.

The functions of mortar are the same in both real and virtual specimens: (1) the volume among skeleton particles is occupied; (2) force applied by adjacent particles is transferred. However, the asphalt sand mortar in simulation does not need to be dense without porosity, and it can occupy the same volume to real condition and has enough contacts with adjacent particles to transfer force.
In order to accurately and efficiently simulate asphalt sand mortar, diameter reduction factor (DRF) was recommended in this research:

$$
\mathrm{DRF}=\frac{D_{i}}{D_{0}}
$$

In equation (1), $D_{0}$ is the initial diameter and $D_{i}$ is the reduced diameter. $D_{0}$ can be a single value $(2.00 \mathrm{~mm}$ for instance) or a range (from $2.00 \mathrm{~mm}$ to $2.36 \mathrm{~mm}$ for instance). In this research, $D_{0}=2.00 \sim 2.36 \mathrm{~mm}$. When DRFs are given, the reduced diameter $\left(D_{i}\right)$ and the volumetric ratio between the two models after and before diameter reduction can be calculated, as shown in Table 2 .

Obviously, with DRFs, the ball element diameters in the equivalent model of asphalt sand mortar can be reduced. As a result, the equivalent model volume is reduced and the particle-interlocking effects can be more realistically simulated.

2.4. DRF-Based Discrete Element Model for Asphalt Mixture. This section presented an improved DEM model of asphalt mixture with the following steps:

(1) Graded clumps and balls are generated simultaneously within a cylindrical sample. According to different application requirements, the height and diameter of the sample may be different. In this paper, both the height and diameter of the cylindrical sample are set as $70 \mathrm{~mm}$. The clumps are generated according to shapes and gradation of coarse aggregates with the method presented in Section 2.2. The balls of diameter from $2.00 \mathrm{~mm}$ to $2.36 \mathrm{~mm}$ are generated with $\mathrm{DRF}=1.0$ according to the method presented in Section 2.3.

(2) Cycling is performed until clump and ball elements become stable. The sample should be compacted until the designed height is met. Note that the design height in this paper is $70 \mathrm{~mm}$ since all the elements are generated according to specimen volume. The compacted sample is the initial sample of asphalt mixture with $\mathrm{DRF}=1.0$. There is a serious overlap in the initial sample due to interference.

(3) Initial sample model will be restored first, and then, different values are assigned to DRF, as shown in Figure 3. Meanwhile, different colours here in Figure 3 also represent different particle size groups. It should be noted that cycling computation should be performed after a new value is assigned to DRF.

(4) The optimum value of DRF should be recommended according to its overlap condition and internal stress to more realistically simulate asphalt sand mortar. Details on the recommendation will be presented in the next section.

\section{Discrete Element Simulation and Diameter Reduction Factor Determination}

As mentioned above, considering the interactions of glued balls in the equivalent model of asphalt sand mortar, it was 


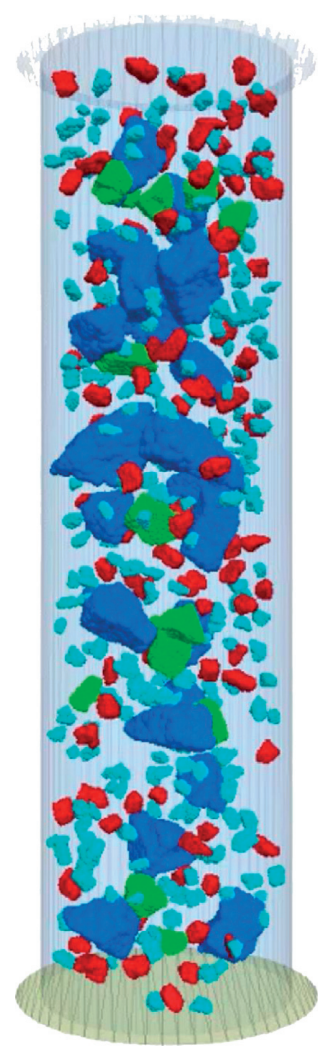

(a)

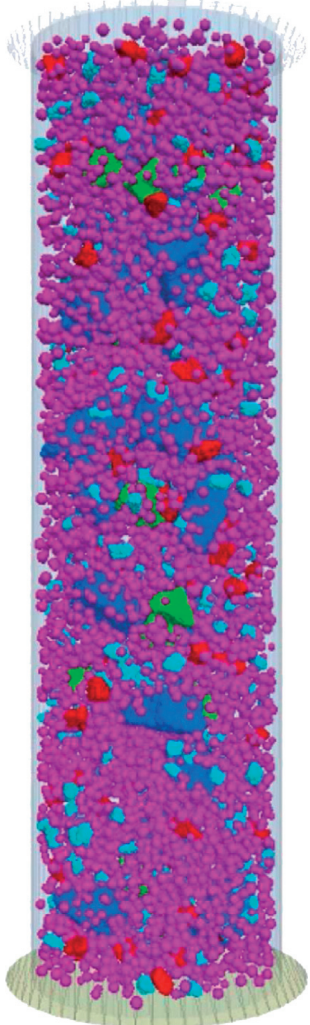

(b)

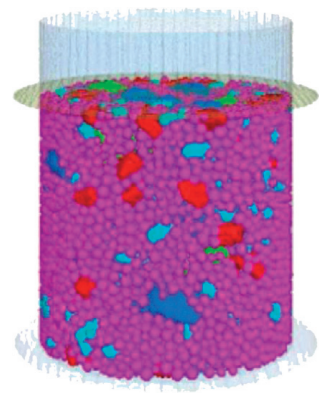

(c)

Figure 2: Clump-based DEM model establishment: (a) coarse particles generation; (b) asphalt sand mortar generation; (c) hydrostatic compaction.

TABLE 1: Micromodelling parameters.

\begin{tabular}{lcc}
\hline Contact types & Friction & Modulus $(\mathrm{GPa})$ \\
\hline Aggregate/aggregate & 0.36 & 55 \\
Aggregate/mortar & 0.20 & 29 \\
Mortar/mortar & 0.20 & 20 \\
Mortar/container & 0.15 & 39 \\
Aggregate/container & 0.15 & 100 \\
\hline
\end{tabular}

necessary to find suitable DRFs for typical asphalt mixtures. In this section, discrete element modelling of typical asphalt mixtures was performed, and results were analysed to determine DRFs with considerations on volumetric overlapping, ball-wall contact number, and interlocking stress within asphalt sand mortar.

\subsection{Gradation and Volumetric Features of Typical Asphalt} Mixtures. As shown in Figure 4, six types of asphalt mixtures were designed based on the Chinese Technical Specification for Construction of Highway Asphalt Pavements JTG F40-2004. To calculate the volume fractions used in virtual models, some assumptions were made as follows based on the existing researches and specifications:

(1) Coarse aggregates, fine aggregates, and fillers come from rocks whose density is assumed to be $2650 \mathrm{~kg} /$ $\mathrm{m}^{3}$.
TABLE 2: Reduced diameters and volumetric ratios of different diameter reduction factors.

\begin{tabular}{ccc}
\hline DRF & $D_{i}(\mathrm{~mm})$ & Volume ratio \\
\hline 1.00 & $2.00-2.36$ & 1.00 \\
0.98 & $1.96-2.31$ & 0.94 \\
0.96 & $1.92-2.27$ & 0.88 \\
0.94 & $1.88-2.22$ & 0.83 \\
0.92 & $1.84-2.17$ & 0.78 \\
0.90 & $1.80-2.12$ & 0.73 \\
0.88 & $1.76-2.08$ & 0.68 \\
0.86 & $1.72-2.03$ & 0.64 \\
0.84 & $1.68-1.98$ & 0.59 \\
0.82 & $1.64-1.94$ & 0.55 \\
0.80 & $1.60-1.89$ & 0.51 \\
0.78 & $1.56-1.84$ & 0.47 \\
0.76 & $1.52-1.79$ & 0.44 \\
0.74 & $1.48-1.75$ & 0.41 \\
0.72 & $1.44-1.70$ & 0.37 \\
0.70 & $1.40-1.65$ & 0.34 \\
0.68 & $1.36-1.60$ & 0.31 \\
\hline
\end{tabular}

(2) The density and content of asphalt binder are assumed to be $1030 \mathrm{~kg} / \mathrm{m}^{3}$ and $5 \%$, respectively.

(3) The air voids of all 6 mixtures were assumed to be $4 \%$. 


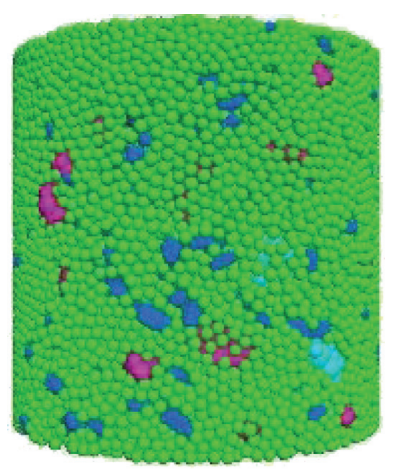

(a)

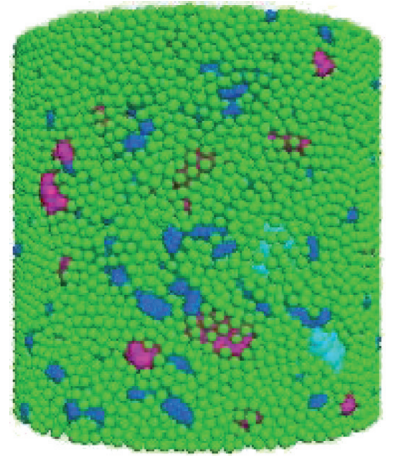

(b)

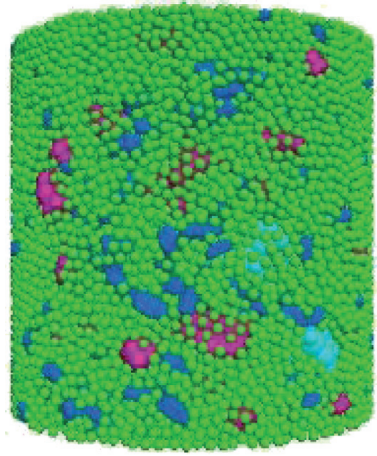

(c)

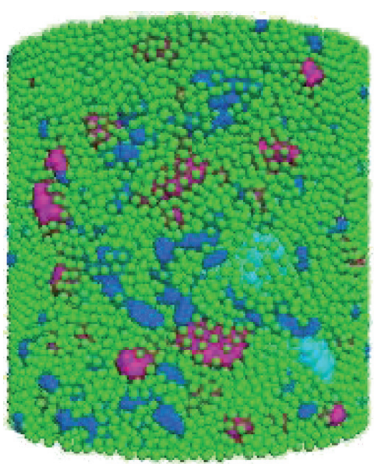

(d)

Figure 3: Discrete element models of asphalt mixture with various DRFs. (a) DRF $=1.00$. (b) DRF $=0.92 .(\mathrm{c}) \mathrm{DRF}=0.84 .(\mathrm{d}) \mathrm{DRF}=0.76$.

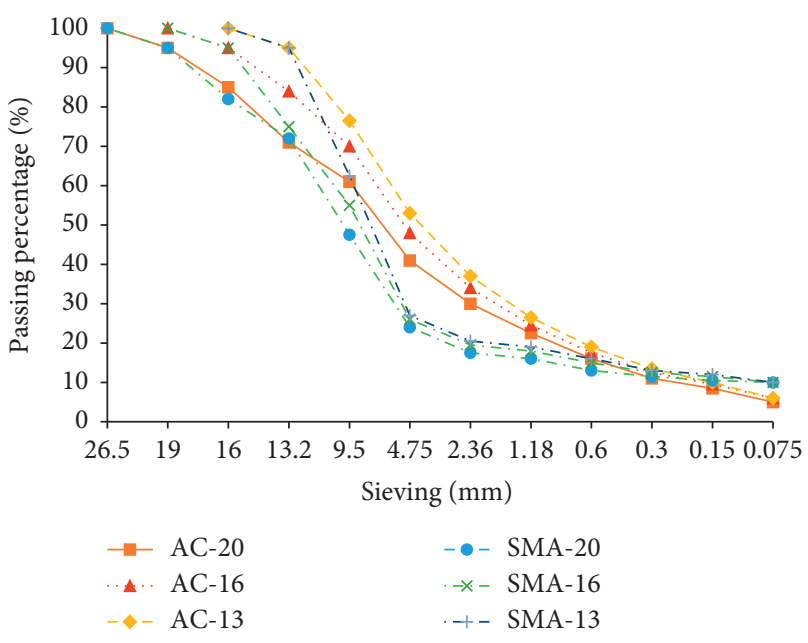

FIgURE 4: Typical gradation curves.

With the information above, the volume fractions of coarse aggregates, asphalt sand mortar (sum of fillers, fine aggregates, and asphalt binder), and air void contents for all 6 gradations are listed in Table 3 . It should be noted that the volumetric parameters used here can be adjusted according to a different situation.

\subsection{Volumetric Overlap Analysis for Determining Diameter} Reduction Factors. Evidently, DRF values represented volumetric features of equivalent models: the decrease in DRF value meant glued ball element diameters were decreasing and then resulted in less overlapping between adjacent discrete elements. In this research, a new concept of "mortar element overlapping ratio" was proposed for determining the rational DRF range.

Figure 5 shows an example to obtain an overlap ratio in PFC. Firstly, the total volume of each mortar element $V_{1}$ was calculated based on their radiuses, as shown in Figure 5(a). Then, the real volume $V_{2}$ can be obtained by the built-in function measure sphere, as shown in Figure 5(b) [18]. Therefore, the overlap ratio can be computed by the following equation:
TABLE 3: Volumetric fractions of mixture components.

\begin{tabular}{lccc}
\hline $\begin{array}{l}\text { Gradation } \\
\text { type }\end{array}$ & $\begin{array}{c}\text { Coarse } \\
\text { aggregates }\end{array}$ & $\begin{array}{c}\text { Asphalt sand } \\
\text { mortar }\end{array}$ & $\begin{array}{c}\text { Air } \\
\text { voids }\end{array}$ \\
\hline AC-13 & 54.18 & 41.82 & 4 \\
AC-16 & 56.76 & 39.24 & 4 \\
AC-20 & 60.20 & 35.80 & 4 \\
SMA-13 & 68.37 & 27.63 & 4 \\
SMA-16 & 69.23 & 26.77 & 4 \\
SMA-20 & 70.95 & 25.05 & 4 \\
\hline \multicolumn{4}{|}{} \\
& overlap ratio $=\frac{V_{1}-V_{2}}{V_{1} .}$
\end{tabular}

As the gradation curves shown in Figure 5 and volumetric fractions listed in Table 2, the discrete element simulation of six typical asphalt mixtures was performed with various DRFs from 0.68 to 1.0 , as listed in Table 1 . A total of 90 discrete element models were built with the method presented in Section 2, while the coarse aggregates were simulated with clumps, and the asphalt sand mortar was simulated with the DRF-based equivalent model. The discrete element simulation data were analysed, and the curves of the mortar element overlap ratio versus DRFs were plotted, as shown in Figure 6.

From Figure 6, it can be observed as follows: (1) with decreasing DRF, the mortar element overlap ratio decreased from about $12 \%$ to less than $0.6 \%$. (2) All 6 gradations had similar curve trends, which firstly linearly decreased to the turning point and then went stabilized. The turning point indicated a critical state that the condition of glued balls started to translate from interlocked to suspended. (3) For AC gradations, the mortar element overlap ratio with DRF values less than 0.80 was almost constant to zero, which meant that 0.80 can be deemed as the optimum value that all glued balls were suspended. (4) SMA gradations were slightly different from AC gradations: curve slopes with DRF values less than 0.76 remained to be nonzero constant. The main reason for this phenomenon was that SMA mixtures had coarser aggregates, and some of the glued balls were interlocking by the strong skeleton and cannot rearrange 


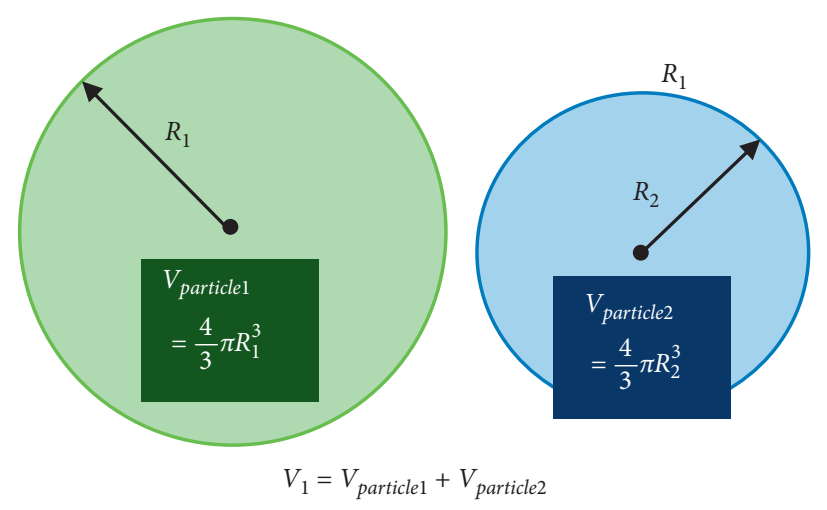

(a)

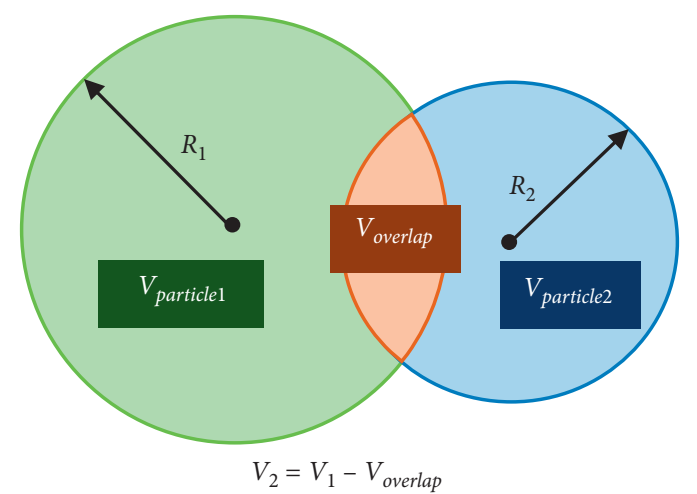

(b)

Figure 5: The calculation of mortar overlap ratio. (a) $V_{1}$ (without overlapping). (b) $V_{2}$ (without overlapping).

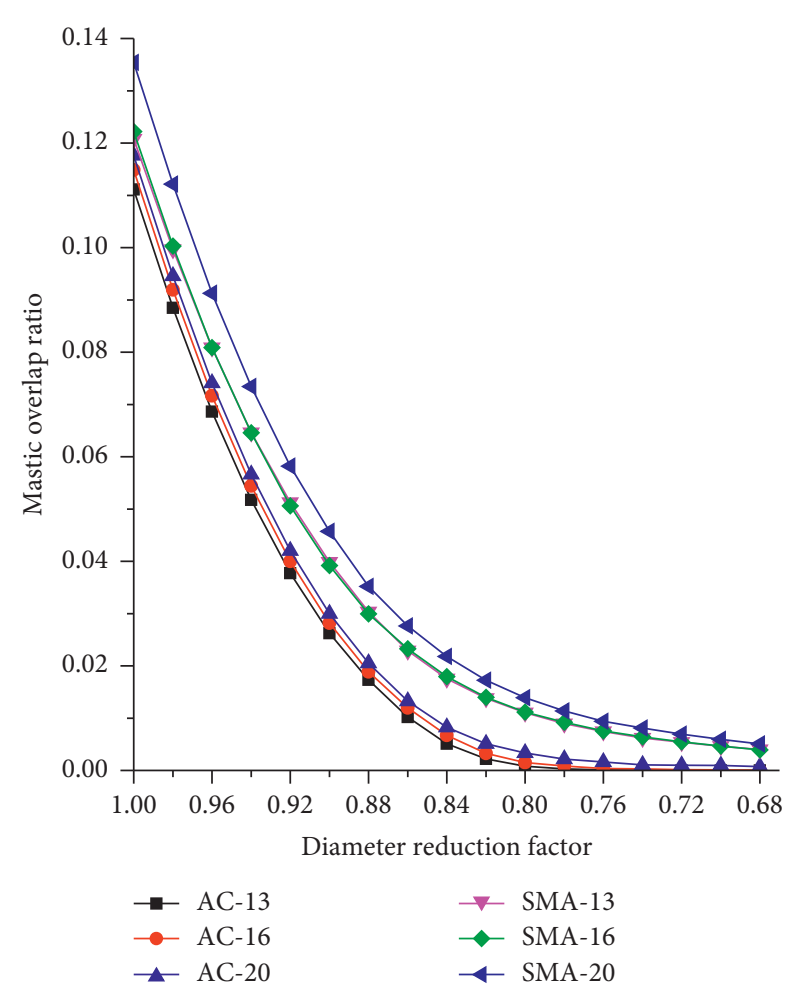

FIGURE 6: Mortar element overlap ratio of AC gradation and SMA gradation.

with a decreasing diameter. Therefore, 0.76 can be deemed as the value that most glued balls were suspended for SMA gradations.

It should be noted that, since increasing nominal diameter resulted in less glued balls volume fraction, the curves tended to entirely move upward from AC gradation to SMA gradation. However, although the glued ball volumes' difference among three AC gradations was larger than those in SMA gradations, AC gradation curves had little difference within each other while had considerable discrepancy compared to SMA gradations, especially when DRF values were less than a turning point. This discrepancy was mainly caused by different skeleton conditions. AC mixtures and SMA mixtures generally had suspended dense structures and skeleton dense structures, respectively. Therefore, it was easier for glued balls in AC mixtures to rearrange with their decreasing diameter. When the mixture's structure is conveyed to a skeleton dense structure, even a small increase in coarse aggregates will result in a stronger skeleton and overlap ratio, which cannot be eliminated by rearrangement. Based on the above analysis, the recommended low limit of DRF could be determined as 0.76 for all mixtures, which meant the glued ball will be in the suspending state if DRF was over the low limit.

3.3. Ball-Wall Contact Number Analysis for Evaluating Diameter Reduction Factor. Similarly, the change in DRF values led to a decreasing ball-wall contact number, which indicated the condition of the ball element changed from an over interlocked state to a suspended state. Therefore, a ballwall contact number was also applied to determine a reasonable DRF range. For Figure 7, six curves displayed how the ball-wall contact number varies with different DRF values of 6 gradation types.

In Figure 7, it can be found as follows: (1) the general tendency of all six curves was that the ball-wall contact number decreased slowly in the initial stage and then dropped dramatically. This phenomenon indicated glued balls were too small to fill the existing voids after and the ball elements tended to be more suspended and even had few contacts with the boundary wall. Hence, the contact numbers were significantly influenced by glued balls volume. (2) The difference between the two gradation types was obvious. The range of curves was from about 4200 to 600 and from 3400 to over 1000 for AC and SMA mixtures, respectively. Meanwhile, curves of AC mixtures descended faster than SMA mixtures due to different mixture structures. (3) In the same gradation type, the mixture with a distinct maximum nominal particle size had a different glued ball volume, which resulted in the discrepancy of three curves in each gradation group. In other words, the more the volume of ball elements, the more the ball-wall contact number, and the faster the decrease rate. 


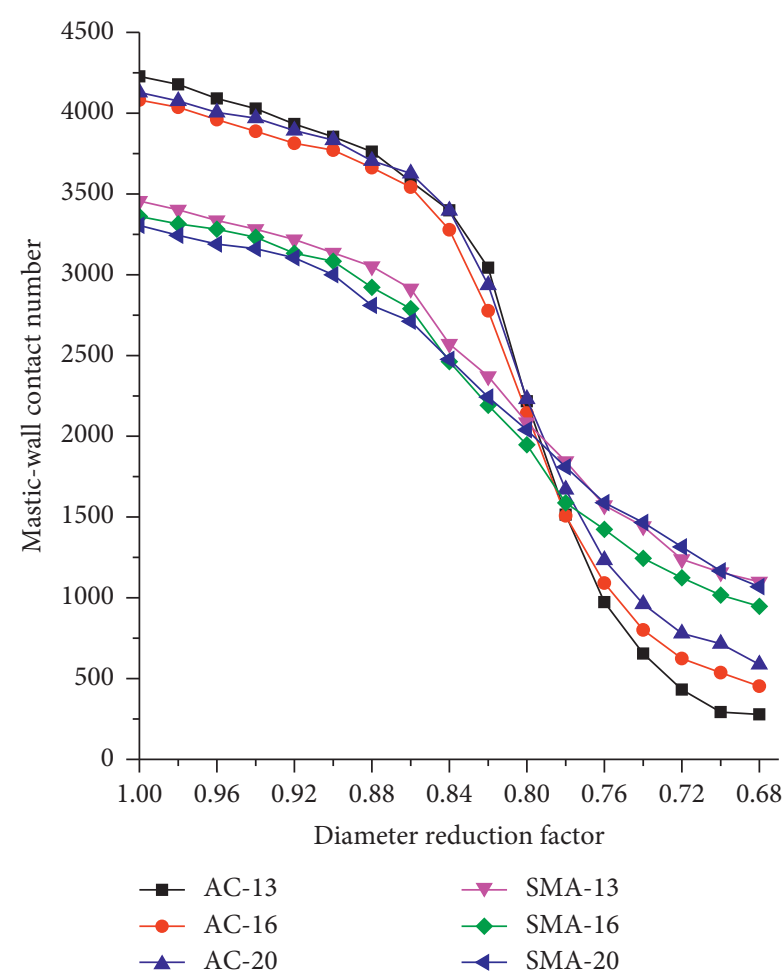

Figure 7: Ball-wall contact number of AC gradation and SMA gradation.

In order to keep glued balls from overpacking, the range of DRF should be determined when the ball-wall contact number started to drop quickly. Therefore, the upper limit of DRF values was chosen as 0.86 for all gradation types.

3.4. Average Wall Stress Analysis for Evaluating Diameter Reduction Factor. Redundant glued balls will not only cause volumetric and structure problems in compacted mixtures but also have a significant impact on the interlocking stress state. Therefore, the average wall stress of every specimen was applied to determine the range of DRF values.

In this model, particles were compacted by the top wall. After the size of the mortar element was reduced according to the DRF value, the internal structure changed and the contact stress between particles and walls also decreased. Through the built-in codes [18], the contact force between wall and particles in the $Z$ direction could be recorded, and the wall stress can be calculated according to the following equation:

$$
\overline{\sigma_{m}}=\frac{\left|F_{t w}\right|+\left|F_{b w}\right|}{\text { wall area }},
$$

where $\overline{\sigma_{m}}$ means the average wall stress and $F_{t w}$ and $F_{b w}$ represent the forces on the top and bottom wall, respectively. And the following figure shows the recorded results of all 6 gradation mixtures.

From Figure 8, it can be observed as follows: (1) curves of average wall stress firstly decreased rapidly, and then, its descending speed slowed down and gradually tended to be stable. The DRF values of the turning point of AC and SMA gradation were about 0.82 and 0.80 , respectively. (2) The

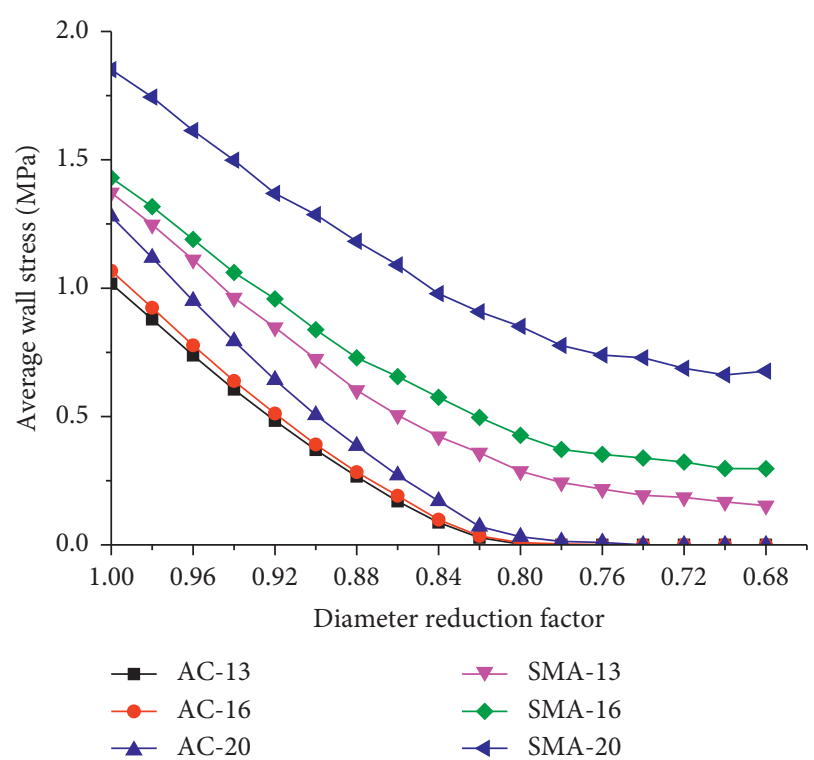

FIgUre 8: Average wall stress of AC gradation and SMA gradation.

average wall stress of AC gradation was less than SMA gradation dramatically due to different structures. (3) The descending speed of AC curves was larger than SMA curves. The reason was that $\mathrm{AC}$ mixtures had more glued balls, and they were more sensitive to the change in DRF values. Note that the determination of the turning point was mainly based on simple observation rather than mathematical methods. The main purpose of this section was to determine a recommended DRF range. Therefore, the turning point was generally selected for convenience. A well-compacted asphalt mixture should have a good interlocking state for all particles. Since the turning point represented the critical state between interlocking and suspending, the optimum DRF value should be bigger than a turning point.

In summary, the analysis of three parameters provided three DRF limits as follows: in Figure 6, DRF should be larger than 0.80 for AC gradation and 0.76 for SMA gradation; in Figure 7, DRF should be less than 0.86 for two gradation types; in Figure 8, DRF should be larger than 0.82 for AC gradation and 0.80 for SMA gradation. Therefore, based on 3 figures, the conservative preliminary DRF ranges were determined as $0.82-0.86$ and $0.80-0.84$ for AC and SMA gradations, respectively. Note that this recommended range could be adjusted according to a different situation.

\section{Application and Verification of Diameter Reduction Factor}

Based on the above analysis, the range of DRF value could be slightly different for different gradation types due to the differences in mortar volume. Some virtual and lab tests were carried out for verifying the recommended DRF value for different gradation types.

4.1. Virtual Compaction Tests. After choosing the midvalue of the recommended gradation range according to 
TABLE 4: Specimen height with different DRFs after virtual static compaction.

\begin{tabular}{lcccc}
\hline DRF & \multicolumn{2}{c}{$\begin{array}{c}\text { Compacted specimen height in DEM } \\
(\mathrm{mm})\end{array}$} & SMA-13 & \multicolumn{2}{c}{$\begin{array}{c}\text { Height difference between DEM and } \\
\text { mixture designed height }(\%)\end{array}$} \\
\hline 0.80 & AC-13 & 68.7 & AC-13 & SMA-13 \\
0.82 & 65.2 & 70.2 & 3.6 & 1.9 \\
0.84 & 67.5 & 71.3 & 0.6 & -0.3 \\
0.86 & 69.6 & 72.7 & -2.7 & -1.9 \\
\hline
\end{tabular}

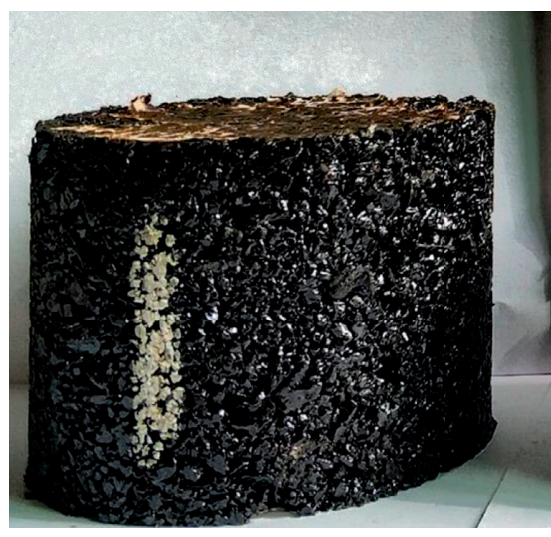

(a)

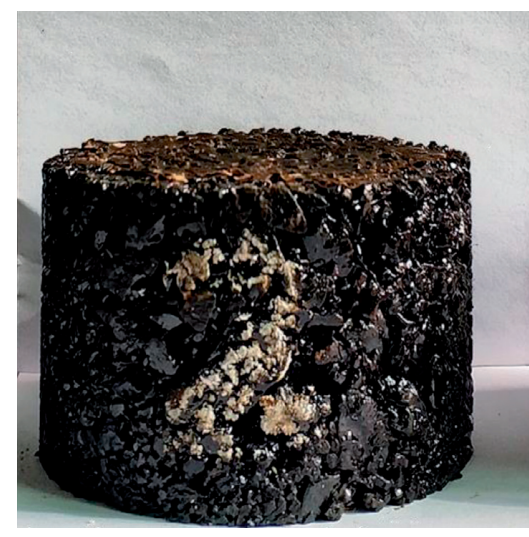

(b)

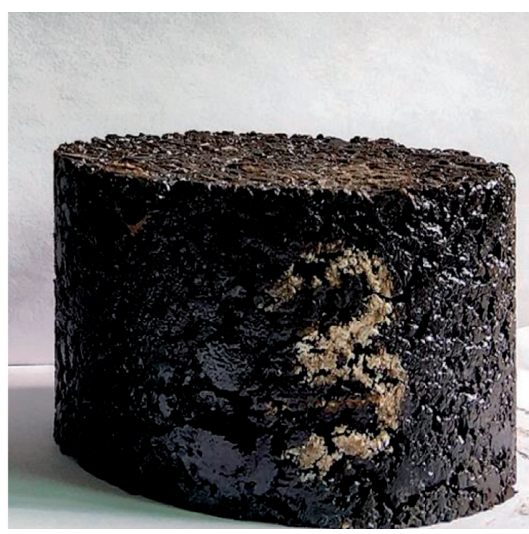

(c)

Figure 9: Specimens with different DRFs after indoor Marshall compaction tests.

Technical Specification for Construction of Highway Asphalt Pavements JTG F40-2004, two gradation mixtures were determined as AC-13 and SMA-13. The general generation and compaction procedures were shown in the following part:

Firstly, according to the above volumetric calculation, the volume of coarse aggregates, fillers, asphalt, and air voids can be obtained. Based on the above analysis, $\mathrm{DRF}$ in the range of $0.80-0.86$ is determined. Hence, there would be 8 specimens generated in this test.

Secondly, coarse-grained aggregates with realistic gradation and particle shapes were generated by clump elements. The asphalt sand mortar was simulated by rigid ball elements with DRF equaling to 1.0 .

Thirdly, the specimens were compacted with a static pressure of $600 \mathrm{kPa}$, and then, the diameters of ball elements were reduced according to different DRFs.

Finally, the compacted specimen heights and height differences between DEM and mixture designed height $(70 \mathrm{~mm})$ were recorded, as shown in Table 4.

According to the height differences between mixture designed height and compacted height, 0.84 is the best DRF value for AC-13 while 0.82 for SMA-13. In fact, the DRF value was mainly determined based on the mortar volume of asphalt mixture, and it can be selected in the range of 0.80-0.86. In addition, the more the mortar volume, the bigger the DRF. The consistency between design height and compacted height also proved that the application of DRF improved the accuracy in volume.

4.2. Indoor Marshall Compaction Tests. Meanwhile, indoor Marshall compaction tests were also applied to verify the recommended DRF value range. It should be noted that the critical variable here was not the compaction methods but the internal relationship of different mixtures. The influence caused by different compaction methods could be negligible in this problem. Therefore, Marshall compaction tests were carried out to verify the simulation results. In this part, there are three compacted specimens with the same coarse gradation (AC-13) as follows: (1) particles less than $2.36 \mathrm{~mm}$ were replaced by fine aggregates in $1.18-2.36 \mathrm{~mm}$ with no volume reduction; (2) particles less than $2.36 \mathrm{~mm}$ were replaced by the fine aggregate in $1.18-2.36 \mathrm{~mm}$ with recommended volume reduction; (3) normal specimen with complete particle sizes. The asphalt content was set to be $5.0 \%$. The final specimens are shown in Figure 9.

After Marshall compaction, the specimens' height is recorded in Table 5:

The following could be observed: (1) without volume reduction, the volume characteristic of the specimen was far from the real situation. (2) The specimen with the recommended volume reduction had a similar height with the specimen in complete gradation. 
TABle 5: Specimen height with different DRFs after indoor Marshall compaction tests.

\begin{tabular}{lcc}
\hline$\# 1$ & $\# 2$ & $\# 3$ \\
\hline $7.236 \mathrm{~cm}$ & $6.247 \mathrm{~cm}$ & $6.309 \mathrm{~cm}$ \\
\hline
\end{tabular}

\section{Summary and Conclusion}

Based on discrete element modelling, this paper generated a virtual specimen of asphalt mixture including aggregate, asphalt mortar, and air voids and then deeply analysed mortar element overlap ratio and average wall stress in different DRFs. During the process, the findings produced are as follows:

After result analysis, the DRF ranges of mortar elements were recommended for different gradations. Given that the initial mortar element diameter was set as $2.00-2.36 \mathrm{~mm}$ for $\mathrm{AC}$ gradation, and the DRF range (0.82-0.86) was recommended, while $(0.80-0.84)$ was chosen for SMA gradation.

The virtual specimen of asphalt mixture was created with real particle shape and gradation, and the recommended generation method of asphalt sand mortar is as follows: (1) calculating the real volume of each part in asphalt mixture specimen; (2)building the basic model with coarse aggregates in the calculated volume; (3) generating mortar ball elements in the size of $2.00 \mathrm{~mm}$ to $2.36 \mathrm{~mm}$ with the initial calculated volume; (4) setting contact models and compacting the mixtures; (5) removing all velocities to make the whole system in a static state; (6) replacing mortar elements with the chosen DRF in the same position.

This article focused on the particle replacement for asphalt mortar. In fact, this method can also be used in other kinds of granular mixtures. The diameter reduction factor can be selected from the recommended range in this article based on fine aggregate volume or conducted a similar virtual test to determine an accurate value.

In conclusion, this article provided a reference for the asphalt mixture simulation in the clump-based discrete element method. The recommended model generation method plays a significant role in DEM further development to solve the problem of asphalt sand mortar dynamic simulation. Both calculation efficiency and enough accuracy were considered in this method, which indicated that DEM could be applied in a more complex situation to deal with more practical problems. However, the current study only focused on solving volumetric problems, and this method still needs to be perfected with consideration of mixture mechanical behaviour. And the in situ replacement method could also be applied to further analysis of asphalt mixtures' gradation in the following papers.

\section{Data Availability}

The data used to support the findings of this study are available from the corresponding author upon request.

\section{Conflicts of Interest}

There are no conflicts of interest regarding the publication of this paper.

\section{Acknowledgments}

This work was supported by the National Natural Science Foundation of China (Grant no. 51978074). The authors also appreciate the funding support from Beijing Key Laboratory of Traffic Engineering, Beijing University of Technology (Grant no. 2018BJUT-JTJD007), and funding support from Beijing Municipal Commission of Transport (Grant no. 40038003201805).

\section{References}

[1] P. A. Cundall and O. D. L. Strack, "A discrete numerical model for granular assemblies," Géotechnique, vol. 29, no. 1, pp. 47-65, 1979.

[2] D. O. Potyondy and P. A. Cundall, "A bonded-particle model for rock," International Journal of Rock Mechanics and Mining Sciences, vol. 41, no. 8, pp. 1329-1364, 2004.

[3] R. Liu, X. Yin, H. Li et al., "Visualization and quantitative profiling of mixing and segregation of granules using synchrotron radiation X-ray microtomography and three dimensional reconstruction," International Journal of Pharmaceutics, vol. 445, no. 1-2, pp. 125-133, 2013.

[4] H. Gao, X. Yang, and C. Zhang, "Experimental and numerical analysis of three-point bending fracture of pre-notched asphalt mixture beam," Construction and Building Materials, vol. 90, pp. 1-10, 2015.

[5] Y. Peng, J.-X. Bao, and Z. Wang, "A comparison of two-dimensional and three-dimensional micromechanical discrete element modeling of the splitting tests for asphalt mixtures," in Proceedings of the 1st International Conference on Transportation Infrastructure and Materials (ICTIM 2016), Xi'an, China, July 2016.

[6] Y. Peng and J.-X. Bao, "Micromechanical analysis of asphaltmixture shear strength using the three-dimensional discrete element method," Journal of Materials in Civil Engineering, vol. 30, no. 11, Article ID 04018302, 2018.

[7] J. Chen, T. Pan, and X. Huang, "Discrete element modeling of asphalt concrete cracking using a user-defined three-dimensional micromechanical approach," Journal of Wuhan University of Technology, vol. 26, no. 6, pp. 1215-1221, 2011.

[8] J. Chen, L.-B. Wang, and X.-M. Huang, "Micromechanical modeling of asphalt concrete fracture using a user-defined three-dimensional discrete element method," Journal of Central South University, vol. 19, no. 12, pp. 3595-3602, 2012.

[9] F. Gong, Y. Liu, X. Zhou, and Z. You, "Lab assessment and discrete element modeling of asphalt mixture during compaction with elongated and flat coarse aggregates," Construction and Building Materials, vol. 182, pp. 573-579, 2018.

[10] X. Ding, T. Ma, and W. Gao, "Morphological characterization and mechanical analysis for coarse aggregate skeleton of asphalt mixture based on discrete-element modeling," Construction and Building Materials, vol. 154, pp. 1048-1061, 2017.

[11] J. Li, J. H. Zhang, G. P. Qian, J. L. Zheng, and Y. Q. Zhang, "Three-dimensional simulation of aggregate and asphalt mixture using parameterized shape and size gradation," 
Journal of Materials in Civil Engineering, vol. 31, no. 3, p. 9, 2019.

[12] J. Chen, B. Huang, and X. Shu, "Air-void distribution analysis of asphalt mixture using discrete element method," Journal of Materials in Civil Engineering, vol. 25, no. 10, pp. 1375-1385, 2013.

[13] D. Zhang, X. Huang, and Y. Zhao, "Algorithms for generating three-dimensional aggregates and asphalt mixture samples by the discrete-element method," Journal of Computing in Civil Engineering, vol. 27, no. 2, pp. 111-117, 2012.

[14] W. Zhou, X. Huang, and L. Wang, "Study on the void reduction behaviour of porous asphalt pavement based on discrete element method," International Journal of Pavement Engineering, vol. 18, no. 4, pp. 285-291, 2017.

[15] J.-F. Ferellec and G. R. McDowell, "A method to model realistic particle shape and inertia in DEM," Granular Matter, vol. 12, no. 5, pp. 459-467, 2010.

[16] X. Zhou, Y. Liu, and Z. You, "Heavy impact compaction modeling and analysis on unbound paving mixtures," in Proceedings of the International Conference on Discrete Element Methods, Dalian, China, August 2016.

[17] F. Gong, X. Zhou, Z. You, Y. Liu, and S. Chen, "Using discrete element models to track movement of coarse aggregates during compaction of asphalt mixture," Construction and Building Materials, vol. 189, pp. 338-351, 2018.

[18] Itasca-Consulting-Group, PFC5.0 Suite Documentation, Itasca-Consulting-Group, Minneapolis, MI, USA, 2014. 